\title{
Prévision des débits et des flux polluants transités par les réseaux d'égouts par temps de pluie. Le modèle FLUPOL
}

\author{
G. Bujon Compagnie générale des eaux, Paris \\ détaché à l'Agence de l'eau Seine-Normandie
}

\begin{abstract}
Les eaux pluviales urbaines sont progressivement devenues une source importante de pollution des cours d'eau récepteurs. L'appréciation de leur impact est facilité par l'emploi des modèles de simulation des eaux pluviales d'une part et de modèles de qualité de rivières d'autre part. FLUPOL a précisément été développé pour simuler les débits et les flux polluants des réseaux d'égout par temps de pluie avec le moins de données possible. La présentation du modèle est suivie d'un exemple de validation grâce à des mesures effectuées dans un égout pluvial de grande taille desservant une vaste région urbanisée au sud de Paris.
\end{abstract}

\section{Introduction}

En raison de l'accroissement constant de l'urbanisation et de l'extension corrélative des surfaces imperméabilisées, le ruissellement pluvial urbain a pris de plus en plus d'importance ces dernières décennies.

Cette importance se manifeste non seulement en matière de débit mais aussi en matière de pollution, celle-ci résultant du lessivage des sols urbains et parfois de l'entraînement de dépôts accumulés dans les conduites des réseaux d'assainissement.

Il faut en effet rappeler que selon la nature du réseau, séparatif ou pluvial, tout ou partie des eaux pluviales éventuellement mélangées à des eaux usées urbaines est rejeté sans épuration dans le milieu naturel, le plus souvent dans des cours d'eau.

Pour juger de l'impact de ces rejets, on peut effectuer des mesures conjointes sur les effluents et dans les cours d'eau récepteurs; mais il est très difficile de réunir au cours de ces mesures des conditions simultanées de fortes pluies et de faible débit de cours d'eau, qui seules sont vraiment intéressantes. En outre, dans des agglomérations de grande taille, le nombre de points de mesure peut être très important. Le recours à la mesure risque ainsi de n'être applicable ni techniquement ni économiquement.
Une autre méthode consiste à mettre au point deux outils de modélisation, l'un pour quantifier les caractéristiques des rejets, l'autre l'impact sur les cours d'eau. Dans ce cas, certaines mesures sur le terrain sont toujours nécessaires mais elles sont bien moins nombreuses car elles restent limitées à l'ajustement et à la validation des modèles.

Le premier de ces outils, qui est décrit dans cet article, a été appelé FLUPOL; il permet de déterminer les caractéristiques des rejets d'eaux pluviales et ceci en relation avec celles des pluies et des bassins de collecte. Il a été conçu pour être utilisé avec un modèle de qualité de rivière dénommé KALPLAN.

En permettant d'évaluer l'impact des eaux pluviales sur les eaux du milieu récepteur, l'utilisation conjuguée de ces deux modèles offre également la possibilité d'étudier les moyens de minimiser les nuisances en testant les répercussions soit d'une gestion différente des ouvrages du réseau d'assainissement, soit de la mise en œuvre d'aménagements susceptibles de modifier les caractéristiques ou le mode de rejet des eaux polluées.

D'une manière plus générale, la conception et l'exploitation de ce type de modèles mathématiques constituent un puissant moyen de compréhension et de clarification des nombreuses relations de cause à effet qui interviennent dans le phénomène global.

Urban stormwater flowrate and quality prediction. The FLUPOL model.

Urban stormwater runoff has become gradually an important source of stream pollution. The use of stormwater simulation and river water quality models helps to appreciate the impact of urban stormwater runoff on the receiving streams. FLUPOL was precisely developed to simulate the quantity and quality response of urban sewer networks with the smallest possible number of data.

The Model presentation is followed by a validation from measurements carried out in a big size storm sewer of a large urban area located south of Paris. 
Ces modèles sont donc non seulement des outils de recherche prévisionnelle, mais aussi des outils très précieux de réflexion.

\section{Considérations sur la conception du modèle FLUPOL}

Il n'est pas nécessaire d'être un spécialiste très averti de l'hydrologie urbaine pour avoir conscience de la complexité et de l'étendue des phénomènes qui interviennent au cours du ruissellement urbain et du transport des eaux pluviales dans un réseau d'assainissement.

Pour simuler par le calcul cette réalité complexe, le technicien peut suivre, a priori, deux cheminements très différents.

Il peut, en se basant sur des résultats de mesures préalables, dégager, grâce à des méthodes d'analyse statistique, les variables explicatives principales puis chercher des relations purement mathématiques permettant de retrouver de manière statistiquement satisfaisante, les grandeurs physiques étudiées à partir de la valeur des variables. Ces relations mathématiques, généralement polynomiales, comportent un nombre plus ou moins important de termes mettant en jeu des fonctions puissance. Les modèles empiriques élaborés selon cette méthode sont parfois appelés, peut-être à tort, modèles statistiques.

Contrairement à cette méthode, ce même technicien peut procéder à une analyse physique, chimique et biologique détaillée des phénomènes qui concourent à la génération et au transport de la pollution d'origine pluviale et modéliser ces phénomènes, à l'aide de lois qui gouvernent le déroulement de chacun d'eux. On effectue ainsi ce qu'il est convenu d'appeler une modélisation déterministe.

Mais on peut douter de la possibilité d'effectuer une modélisation déterministe de la totalité des phénomènes, en décrivant mathématiquement chacune des relations de cause à effet qui interviennent dans leur déroulement.

On peut, à défaut, imaginer une description plus ou moins globale d'un ensemble de phénomènes, à l'aide d'un nombre restreint de paramètres particulièrement explicatifs, que des études de terrain préalables ont permis de dégager. Certains parlent à ce sujet de modélisation conceptuelle.

On obtient ainsi des modèles spatio-temporels faisant intervenir des coefficients dont la valeur peut dépendre de celle de plusieurs paramètres et dont la loi de variation peut être dégagée grâce à des mesures préalables.

Ainsi, quelle que soit la voie choisie, la modélisation passe par une ou plusieurs campagnes de mesures, ayant pour but de faire apparaître les principaux paramètres explicatifs et de déterminer les coefficients numériques dont il faut les affecter pour que les modèles rendent compte de la réalité d'une manière satisfaisante. Cette détermination est généralement appelée calage du modèle.

Cependant l'expérience montre que le calage des modèles statistiques est très souvent spécifique du site des mesures, contrairement à celui des modèles à ca- ractère déterministe ou conceptuel; en d'autres termes, un modèle déterministe ou conceptuel calé sur un site peut souvent être utilisé tel quel sur d'autres sites sans entraîner de grosses erreurs d'estimation, ce qui n'est généralement pas le cas d'un modèle statistique au sens précisé ci-avant.

C'est ce qui nous a fait préférer une modélisation de type conceptuel, compte tenu du type d'application envisagé pour le modèle, de l'étendue de la région sur laquelle doit porter la modélisation et de l'impossibilité de réaliser un calage dans toutes les parties de cette région.

L'établissement du modèle a été guidé par deux principales préoccupations (non totalement indépendantes l'une de l'autre).

La première visait la plus grande simplicité possible compatible avec une précision acceptable du résultat.

La seconde avait pour objet le respect d'une certaine homogénéité entre les différents modèles élémentaires décrivant les divers phénomènes en cause. Il paraissait illusoire en effet, de décrire avec une très grande précision certains de ces phénomènes, alors que d'autres, plus complexes ou moins bien connus, ne pouvaient faire l'objet que d'une description bien plus sommaire.

Cette préoccupation a d'ailleurs été étendue à la conception du modèle KALPLAN précité, les deux modèles devant, dans notre esprit, constituer un tout cohérent.

\section{Principe du calcul des débits et des flux polluants}

Par débits, on entend, bien évidemment, les débits d'eaux pluviales et éventuellement les débits d'eaux usées de temps sec, et par flux polluants les flux de matières en suspension totales ou décantables, de DBO5, de DCO et de matières azotées. (Azote Kjeldhal : azote organique et ammoniacal encore appelé Azote total Kjeldhal-NTK).

Entre deux pluies successives, des déchets urbains de toutes sortes s'accumulent sur le sol des agglomérations. Dans le même temps des dépôts sont susceptibles de se former dans certaines conduites des réseaux d'égouts par lesquels transitent également des eaux usées urbaines.

Lorsque survient la pluie, celle-ci ruisselle sur le sol; sous l'effet du choc des gouttes d'eau et des forces de frottement du ruissellement, de fines particules solides plus ou moins polluées de matières organiques sont entraînées par l'eau en mouvement. Parvenues dans le réseau d'égouts après un cheminement complexe les eaux pluviales y induisent des écoulements transitoires au cours duquel peuvent se produire des décantations de particules solides ou au contraire des remises en suspension de particules déposées précédemment.

C'est principalement la succession de tous ces phénomènes que le modèle FLUPOL permet de simuler d'une manière plus ou moins globale.

La mise en œuvre du modèle suppose une schématisation préalable du réseau obtenue en ne retenant que les collecteurs principaux.

Ces collecteurs sont eux-mêmes divisés en tronçons de caractéristiques homogènes (dimensions, pente...) en 
tenant compte de surcroît d'un certain nombre de points singuliers (confluences de deux collecteurs, dérivations, déversoirs) qui seront examinés plus bas.

Pour chacun des points particuliers ainsi définis, on détermine la zone de collecte correspondante et on vérifie que cette zone présente des caractéristiques homogènes.

Si ce n'est pas le cas, on procède à une subdivision en autant de zones homogènes nécessaires, ce qui conduit à déterminer les exutoires respectifs dans le réseau, donc à une subdivision des tronçons précédem. ment définis.

Chaque point de séparation entre tronçons ou éléments de tronçon va constituer un point de calcul de débit ou de flux polluant. Ces points peuvent être :

- des points " tête " qui sont à l'origine des collecteurs; - des points "déversoirs" d'où sont dérivés, au-dessus d'un seuil de cote souvent réglable, des rejets vers le milieu naturel;

- des points "dérivation/relai" d'où sont dérivés, autrement que par un seuil, des débits vers un collecteur voisin ou qui sont tout simplement des points de calcul intermédiaires, motivés par une particularité quelconque de la zone de desserte ou du collecteur qui la dessert. Aux points "relai" les débits et flux dérivés sont naturellement fixés à zéro.

- des points " confluence " où convergent les effluents de deux collecteurs principaux, les débits et les flux de chacun d'eux étant calculés au cours de la même séance de travail.

- des points "Affluents" où le collecteur étudié reçoit des effluents provenant d'un collecteur voisin, le débit et le flux de ceux-ci étant une donnée de calcul déterminée au cours d'une autre séance préalable de calcul.

Le calcul des débits et des flux est effectué point par point de l'amont vers l'aval. Dans le cas des réseaux séparatifs ce sont ceux des seules eaux pluviales, dans le cas des réseaux unitaires on leur ajoute les débits et les flux des eaux usées de temps sec en prenant, au besoin, les valeurs de pointe quotidienne pour se placer dans les conditions les plus défavorables.

Un pas de temps convenable ayant été choisi (quel. ques minutes), on effectue, en chaque point et pour chaque pas, un bilan des débits et flux entrants et sortants, puis on propage débits et flux résultants jusqu'à l'amont immédiat du point suivant.

Les débits et flux entrants comprennent les apports de la partie de zone de collecte desservie par le tronçon de collecteur entre le point considéré et le point immédiatement à l'amont, les débits et les flux propagés en provenance de ce dernier et éventuellement, pour des points confluence ou affluent, les débits et flux reçus d'un autre collecteur.

Les débits et flux sortants sont les débits passant au-dessus d'un seuil déversoir ou dérivés vers un autre collecteur, ainsi que les débits et flux dirigés vers le point de calcul suivant.

Les phénomènes éventuels de dépôt ou de remise en suspension des matières solides dans le collecteur, sont pris en compte dans l'établissement des bilans précités.

Entre deux points successifs de calcul, le collecteur est supposé avoir des caractéristiques constantes, tant en

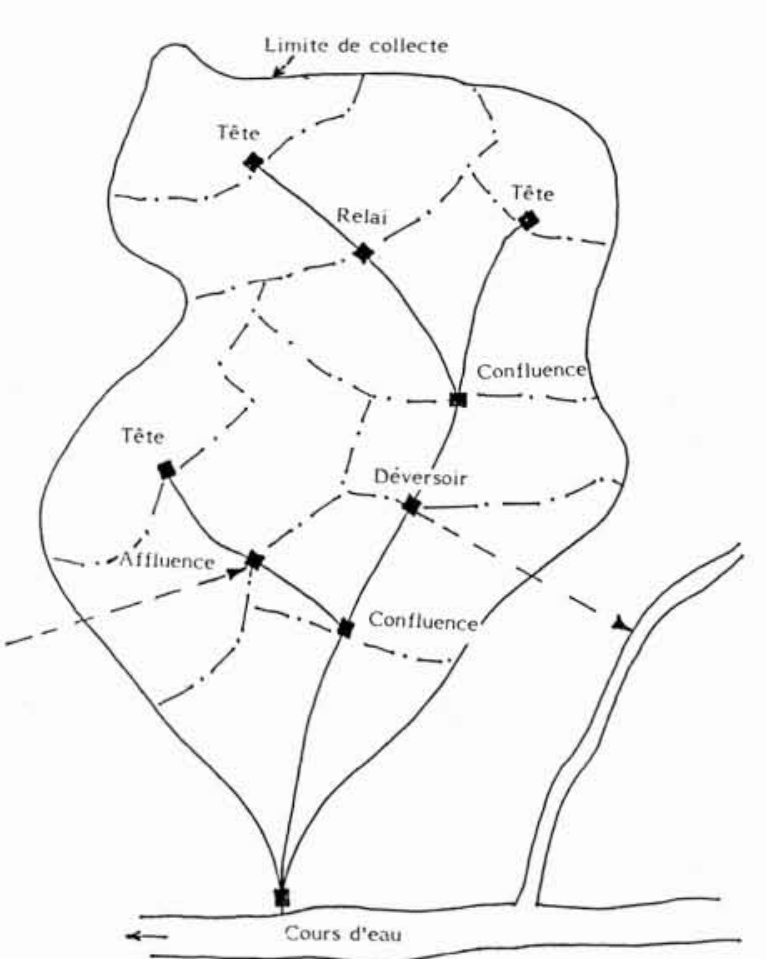

1. Exemple de zone de collecte schématisée en vue d'une simula. tion.

- Point de calcul

ce qui concerne sa section droite et sa pente, que sa rugosité. On suppose en outre qu'il n'y a aucun apport de débit ou de flux sur le tronçon du collecteur en cause, les apports de la partie de zone de collecte desservie étant localisés juste à l'amont du point de calcul.

Le calcul des apports et des pollutions remises en suspension nécessite la connaissance de l'état de salis. sure du sol et des dépôts sur le fond du réseau d'assainissement en début de pluie.

Ces données dépendent elles-mêmes des périodes de temps sec et des événements pluvieux qui ont précédé la pluie en cause. On ne peut les connaître qu'en effectuant une simulation continue à partir d'un événement antérieur suffisamment intense et en supposant qu'il ne restait ni sallissure sur le sol ni dépôt sur le fond des conduites à l'issue de cet événement.

Une procédure de calcul accéléré a été prévue de manière à ce que cette simulation continue n'allonge pas exagérément le temps de calcul.

La figure 1 présente un exemple de zone de collecte schématisée en vue d'une opération de modélisation.

Un programme de calcul informatisé a été mis au point pour effectuer rapidement les très nombreux calculs qu'il faut exécuter au cours d'une simulation. Ce programme de calcul qui a été écrit en langage Basic occupe 34300 octets; il peut évidemment être exécuté à l'aide d'un classique interpréteur Basic mais pour simuler le fonctionnement de réseaux tant soit peu complexes, il est pratiquement indispensable, pour que les temps de calcul ne soient pas prohibitifs, de compiler le programme préalablement à son exécution. 


\section{Principaux algorithmes du modèle}

\section{Calcul des apports}

Pour calculer les débits d'eaux pluviales, on a retenu un modèle de transformation pluie débit classique dit modèle de réservoir linéaire qui traduit qu'à un instant donné, existe une proportionnalité entre le volume d'eau $S$ stocké dans une zone de collecte et le débit $Q$ qui est évacué à la sortie de celle-ci.

On a donc :

$$
S(t)=K \cdot Q(t) \quad \text { d'où } \quad d S=K \cdot d Q
$$

Mais si $i(t)$ est l'intensité de la pluie au temps $t$ et que le débit est rapporté à la surface du bassin, grandeur qui est homogène à une hauteur d'eau par unité de temps, on peut écrire l'équation de continuité ci-dessous :

$$
d S=(i(t)-Q(t)) d t
$$

il s'ensuit

$$
K \frac{d Q}{d t}+Q(t)=i(t)
$$

En définitive, le débit peut être calculé par l'expression :

$$
Q=Q_{i} \cdot e^{-\imath / K}+\frac{1}{K} \int_{0}^{T} e^{-(t-u) / K} \cdot i(u) \cdot d u
$$

dans laquelle:

$u$ est le temps courant depuis le début de la pluie;

$t$ le temps pour lequel on calcule le débit,

$T$ la durée de la pluie si $t$ est égal ou plus grand que cette durée,

ou le temps de simulation $t$, si celui-ci est plus petit ou égal à la durée de la pluie,

$Q_{i}$ le débit résiduel éventuel résultant d'une pluie précédente.

On peut trouver une solution analytique à la relation (4) dans le cas de pluies de projet dont l'hyétogramme présente une forme géométrique simple. Pour des pluies réelles, il faut procéder à une intégration numérique. $\mathrm{Si}$ $\Delta t$ est le pas de temps choisi et $i(N)$ l'intensité de la pluie au pas $N$ supposée constante au cours de chaque pas, le débit au pas $N$ s'écrit:

$$
Q_{(N)}=Q_{(N-1)} e^{-\Delta t / K}+\left(1-e^{-\Delta t / K}\right) \cdot i(N)
$$

Ce modèle est très simple mais il soulève le problème du choix de valeur du coefficient de proportionnalité $K$. Ce coefficient dépend d'évidence des caractéristiques de la zone de collecte, de celles du collecteur qui la dessert et des caractéristiques de la pluie. Dans une très belle étude de ce modèle, M. Desbordes [1] a proposé une expression qui, avec les unités indiquées dans la liste des symboles, se présente de la manière suivante :

$$
\begin{aligned}
\mathrm{K}= & 79,35 \mathrm{AR}^{0,18} \cdot \mathrm{PNT}^{-0,36} \cdot \\
& (1-\mathrm{IMPR})^{-1,9} \cdot \mathrm{T}_{3}^{0,21} \cdot \mathrm{LNG}^{0,15} \cdot \mathrm{HPE}^{-0,07}
\end{aligned}
$$

Dans laquelle :

AR superficie de la zone desservie;

PNT pente moyenne du terrain;

IMPR coefficient d'imperméabilisation;

T3 durée de la pluie;
LNG longueur maximale de la zone;

HPE hauteur d'eau précipitée.

Le modèle n'est donc pas réellement linéaire et d'ailleurs une exploitation fine des résultats de mesure permet de constater qu'au cours d'une pluie la grandeur $S(t)=f(Q)$ n'évolue pas tout à fait sur une droite mais décrit une boucle, généralement très aplatie, que l'on peut presque toujours, en zone urbaine, assimiler à une droite de pente $K$. C'est pourquoi il n'a pas paru utile d'envisager une variation de $K$ au cours de chaque événement pluvieux, compte tenu par ailleurs des complications de modélisation inévitables qui s'ensuivraient et des résultats satisfaisants obtenus avec le modèle pseudo-linéaire adopté.

Il n'est pas certain toutefois qu'il ne faudrait pas adopter quelques correctifs dans le cas de pluies exceptionnellement fortes.

Selon le réseau d'égout considéré, il peut y avoir un débit de temps sec que l'on simule à l'aide de l'expression ci-dessous :

$$
Q \mathrm{SEC}=\mathrm{FR} \cdot \mathrm{PTE} \cdot \mathrm{AR} \cdot \mathrm{DENS} \cdot \mathrm{QU} / 86400
$$

avec FR fraction de population raccordée;

PTE coefficient de pointe horaire;

DENS densité de population de la zone;

QU rejet spécifique d'eau usée $\left(\mathrm{m}^{3} / \mathrm{J} /\right.$ habitant)

En ce qui concerne les apports polluants, on dissocie le phénomène physique en trois phases.

La première correspond au dépôt des polluants sur le sol au cours du temps entre la fin de la pluie précédente et le début de la pluie étudiée.

La seconde traduit la libération des polluants accumulés sur le sol sous l'effet de l'intensité de la pluie; on pourrait également qualifier cette seconde phase de mise en solution ou en suspension des polluants dans l'eau de ruissellement.

La troisième a trait à l'évacuation des polluants en solution ou en suspension, à l'éxutoire de la partie de zone de collecte desservie par le point.

La manière la plus simple de décrire la phase d'accumulation consiste à supposer un accroissement linéaire des poussières et salissures déposées sur le sol au cours du temps. L'expérience a cependant montré qu'une telle description entraîne une surestimation des masses polluantes présentes sur le sol; on est ainsi conduit à imaginer une limitation à la croissance linéaire.

Après plusieurs essais, on a adopté l'expression suivante pour modéliser la variation dans le temps de la masse de polluants solides au sol:

avec :

$$
\frac{\mathrm{dM}}{d t}=P S-K_{1} M
$$

$M$ masse déposée à l'instant $t$

$P S$ quantité de poussières et salissures se déposant sur le sol dans l'unité de temps et d'espace

$K_{1}$ coefficient de "disparition" des polluants, ce coefficient pouvant traduire, par exemple, l'effet d'une dégradation biologique ou du nettoyage de la voierie.

Si $M R$ est la masse résiduelle de dépôts à la fin de la pluie précédente, la masse $M O$ présente au sol au début 
de la pluie suivante, après une période de temps sec $t$, est donnée par l'expression ci-dessous, qui résulte de l'intégration de l'équation (8)

$$
\mathrm{MO}=\mathrm{MR} \cdot e^{-K_{1} t}+\frac{\mathrm{PS}}{K 1}\left(1-e^{-K_{1} t}\right)
$$

La quantité spécifique de dépôt $P S$ dépend naturellement du mode d'occupation du sol. Les premières simulations effectuées l'ont été à l'aide de valeurs trouvées dans la bibliographie U.S. où l'on distinguait quatre types d'occupation, résidentiel dense, résidentiel discontinu, commercial et industriel et où les valeurs de $P S$ étaient données en masse déposée par jour et par unité de longueur de trottoir. Transposées en masse déposée par jour et par hectare, ces valeurs de $P S$ deviennent approximativement :

$\begin{array}{lrl}\text { zone résidentielle dense } & 10 \mathrm{~kg} / \mathrm{j} / \mathrm{ha} \\ \text { zone résidentielle discontinue } & 5 & \text { " } \\ \text { zone commerciale } & 15 & \text { " } \\ \text { zone industrielle } & 35 \mathrm{"}\end{array}$

Si l'on excepte le cas des zones industrielles pour lesquelles on ne dispose pas de résultats expérimentaux particuliers et où d'ailleurs les valeurs de $P S$ devraient dépendre fortement des industries représentées, il est apparu que les valeurs indiquées ci-avant, sont en ordre de grandeur en accord avec les résultats expérimentaux disponibles. Mais ce jugement dépend bien entendu de la valeur du coefficient de disparition $K 1$ retenu après calage, de l'ordre de $0,08 \mathrm{j}^{-1}$ et il conviendra donc d'affiner toutes les valeurs provisoires actuelles à l'aide de résultats de mesures à venir.

Pour ce qui est de la mise en solution ou en suspension des pollutions dans l'eau de ruissellement sous l'effet de la pluie, on a admis que la vitesse du phénomène est proportionnelle à la quantité disponible au sol

$$
\frac{d M}{d t}=-K_{A} \cdot M
$$

le coefficient de proportionnalité $K_{A}$ dépendant de l'intensité $i(t)$ de la pluie. Ainsi que l'ont proposé les auteurs du modèle U.S. bien connu des spécialistes, le Storm Water Management Model (SWMM), cette intensité étant exprimée en $\mathrm{m} / \mathrm{h}$ on $\mathrm{a}$ :

$$
K_{A}=181,3 \cdot i(t)
$$

L'évolution de la masse au sol peut donc s'obtenir très simplement par intégration de (10) et la masse résiduelle $M R$ au temps $t$, l'intensité de la pluie étant supposée constante, est donnée par:

$$
M R=M O \cdot e^{-K_{A} t}
$$

Sous forme discrétisée, telle qu'on l'utilise généralement, cette relation s'écrit :

$$
M R=M R O \cdot e^{-K_{A} \cdot \Delta t}
$$

ou encore

$$
M R O-M R=M R O\left(1-e^{-K_{A} \cdot \Delta T}\right)
$$

$M R O$ - $M R$ représentant la masse entraînée pendant le pas de temps $N$ de durée $\Delta t$.

Mais les simulations réalisées à l'aide de cette relation ont mis en évidence une surévaluation des masses entraînées pour les faibles intensités de pluie et au contraire une sous-estimation pour les intensités élevées. Ces défauts ont pu être corrigés, par les auteurs du SWMM, en appliquant au résultat un coefficient de correction $A$ lui-même fonction de l'intensité instantanée de la pluie :

$$
\begin{gathered}
A=0,057+651619 i_{(\aleph)}^{1,1} \\
(i \text { en } \mathrm{m} / \mathrm{h})
\end{gathered}
$$

et la formule (13) devient ainsi après correction :

$$
M R O-M R=A \cdot M R O\left(1-e^{-K_{A} \cdot \Delta \eta}\right)
$$

$K_{A}$ et $A$ étant donnés respectivement par les relations (11) et (15)

ou encore :

$$
M R=M R O\left[1-A\left(1-e^{-K_{A} \cdot \Delta t}\right)\right]
$$

On peut remarquer que l'intensité de la pluie intervient à la fois dans le terme $K A$ de la relation différentielle (10) et dans le terme $A$ des relations intégrées (16) et (17). On peut ainsi se demander quelle serait la forme d'un terme $K A^{\prime}$ ', s'il existait, qui dans l'expression $d M / d t=-K A^{\prime} \cdot M$, semblable à l'équation différentielle de départ, conduirait aux mêmes effets cumulés que $K A$ et $A$.

On ne peut répondre précisément à cette question car, en fait, il n'existe pas de $K A^{\prime}$ exactement équivalent; mais le calcul montre que pour des pas de temps $\Delta t$ suffisamment petits, on peut assimiler $K A^{\prime}$ au produit $A . K A$, ce qui, tous calculs effectués, conduirait par conséquent à :

$$
K A^{\prime}=118,1385 \cdot 10^{6} \cdot i_{(N)}^{21}+10,33 \cdot i_{(N)}
$$

On voit ainsi que l'intensité de la pluie intervient sensiblement par son carré.

Il s'agit maintenant de déterminer le flux de pollution qui est évacué à l'exutoire de la partie de zone de collecte en cause. Il a été supposé pour celà qu'il existe une relation de proportionnalité entre la masse polluante $M e(t)$ présente en suspension ou en solution, au temps $t$ et le flux $F(t)$ sortant au même instant. Celà s'exprime par:

$M e(t)=K \cdot F(t) \quad$ où $\quad d M e(t)=K \cdot d F(t)$

Le choix du coefficient de proportionnalité $K$ sera explicité plus bas.

Mais on peut aussi écrire l'équation de continuité suivante :

$$
d M e(t)=[\phi e(t)-F(t)] \cdot d t
$$

qui exprime que la variation de masse polluante en suspension ou en solution dans le temps élémentaire $d t$ est égale à la différence des flux polluants mis en suspension ou en solution par la pluie $\phi e(t)$ et évacué de la zone étudiée $F(t)$

Dans ces conditions :

$$
K \frac{d F(t)}{d t}+F(t)=\phi e(t)
$$

Il s'ensuit que le flux cherché est donné par:

$$
F(t)=F_{i} e^{-t / K}+\frac{1}{K} \int_{0}^{T} e^{-(t-u) / K} \cdot \phi e(u) \cdot d u
$$


Très généralement $F(t)$ doit être calculé numériquement; $\Delta t$ étant le pas de temps choisi et $\phi e(N)$ le flux polluant entraîné dans la zone de collecte au pas $N$, le flux évacué de cette zone s'écrit:

$$
F_{(N)}=F_{(N-1)} e^{-\Delta t / k}+\left(1-e^{-\Delta t / k}\right) \cdot \phi e(N)
$$

avec

$$
\phi e(N)=\frac{M R-M R O}{\Delta t}+\frac{P S}{86400}
$$

On a donné au coefficient de proportionnalité $K$ dés expressions (19) à (23) la même valeur que celle donnée au coefficient de l'expression (1); dans les deux cas cette valeur est donc calculée grâce à la relation (6).

La pollution dont il a été question jusqu'ici est constituée de poussières et salissures qui se retrouvent dans l'effluent de la zone de collecte sous la forme de matières en suspension.

Ces poussières et salissures présentent certaines caractéristiques de DBO5, DCO et d'azote total KJELDHAL dont il est également utile de connaître les flux.

Il avait été considéré au départ que ces divers flux pouvaient être pris proportionnels au flux de matières en suspension, le coefficient de proportionnalité $K P$ étant constant. Les résultats de mesure dont on a pu disposer par la suite ont montré l'inexactitude de cette hypothèse et ont conduit à retenir un coefficient de proportionnalité variable, fonction de l'intensité maximale de la pluie (sur $5 \mathrm{mn}$ ) atteinte au cours de l'événement donnant lieu à modélisation.

La relation donnant ce coefficient est de la forme :

$$
K P=a \cdot I_{\text {Max }}^{b}+d
$$

$a, b$ et $d$ étant des coefficients numériques et les relations étant différentes pour chacun des trois paramètres à simuler. De cette façon, le coefficient $K P$ reste constant pour un événement donné mais peut varier d'un événement à l'autre. Il aurait pu être envisagé de faire varier $K P$ à chaque pas de temps mais compte tenu des résultats de simulation satisfaisants obtenus jusqu'à présent, cette option n'a toujours pas été retenue.

Pour la simulation éventuelle des flux de temps sec, on se place, ici encore, dans les conditions de flux de pointe et on applique la relation très simple.

$$
\text { FSEC }=\text { FR.PTE.AR.DENS.GP/86400 }
$$

FR, PTE, AR et DENS ont déjà été définis précédem. ment; GP est le rejet spécifique de pollution, à savoir :

$\begin{array}{lll}\text { MES } & 0,09 & \mathrm{~kg} / \mathrm{j} / \text { habitant } \\ \text { DBO5 } & 0,054 \mathrm{~kg} / \mathrm{j} / \text { habitant } \\ \text { DCO } & 0,1 \quad \mathrm{~kg} / \mathrm{j} / \text { habitant } \\ \text { N } & 0,011 \mathrm{~kg} / \mathrm{j} / \text { habitant. }\end{array}$

\section{Propagation des hydrogrammes}

Après avoir effectué, en chaque point de calcul et pour chaque pas de temps, un bilan des débits, on dispose d'hydrogrammes qu'il convient de propager jusqu'à l'amont du point de calcul suivant.

C'est là un problème bien connu des hydrauliciens qui proposent toute une gamme de solutions de la plus complète, mais aussi la plus complexe basée sur l'intégration des équations de Saint Venant, à la plus simple qui consiste en une translation de l'hydrogramme à vitesse constante.

Dans le cas présent, et compte tenu des idées développées au paragraphe 2 , deux méthodes de propagation ont été prévues.

La première dite de l'onde cinématique néglige les phénomènes d'inertie et suppose qu'il existe une relation biunivoque entre débit et tirant d'eau ou encore entre débit et section de l'écoulement. Ce cas correspond à la propagation sans déformation de l'hydrogramme calculé au départ du tronçon de collecteur considéré, la vitesse de propagation étant la célérité de l'onde cinématique supposée constante. En d'autres termes, l'hydrogramme à l'aval du tronçon est obtenu en décalant l'hydrogramme calculé en tête de celui-ci d'un temps correspondant à la durée de déplacement de l'onde de débit entre les deux points.

Dans le cas d'une conduite de section rectangulaire, la vitesse de déplacement $c$ peut être estimée grâce à la relation ci-dessous :

$$
c=\frac{5}{3} V I T\left[1-\frac{4}{5} \frac{1}{2+L / H}\right]
$$

$V I T$ étant la vitesse moyenne d'écoulement, $L$ et $H$ étant respectivement la largeur et la hauteur de celui-ci.

Dans le cas d'une conduite de section circulaire, les caractéristiques de l'écoulement sont conditionnées par l'existence des dépôts éventuels. Si $D I$ est le diamètre de la conduite, $S E$ la surface transversale à l'écoulement occupée par l'eau, $S A F$ celle occupée par les dépôts et $L D E P$ la largeur supérieure de ces derniers, la vitesse de déplacement de l'onde de débit est calculée par l'expression :

$$
c=\frac{5}{3} V I T[1-W A / W B]
$$

VIT étant la vitesse moyenne de l'écoulement de l'eau. avec

$$
\begin{gathered}
W A=S E\left(\cos \frac{8(S E+S A F)}{D I^{2}}+\frac{2}{D I}\right) \\
W B=7\left(0,25 D I \cdot \sin \frac{8(S E+S A F)}{D I^{2}}+4 \frac{S E}{D I}+L D E P\right)
\end{gathered}
$$

La vitesse moyenne d'écoulement VIT est elle même une vitesse pondérée par rapport au débit donnée par :

$$
V I T=\frac{\Sigma V_{N} \cdot Q_{N}}{\Sigma Q_{N}}
$$

où $Q_{N}$ est le débit moyen de l'hydrogramme au nième pas de temps et $V_{N}$ la vitesse d'écoulement du régime uniforme correspondant à $Q_{N}$.

Mis à part le problème éventuel que pose, dans le cas des conduites circulaires, le calcul de la surface transversale de dépôts $S A F$, problème qui sera examiné plus loin, cette méthode de propagation est la plus simple que l'on puisse concevoir et elle donne généralement de très bons résultats. 
Il y a cependant des exceptions lorsque pour un débit donné, la pente des conduites est trop faible ou lorsque la longueur du tronçon sur laquelle le débit est propagé est trop grand. Dans ce cas en effet on constate un amortissement des pointes de débit que ne peut naturellement pas simuler la méthode de l'onde cinématique.

Il faut alors faire appel à un autre modèle de propagation. Dans un premier temps on a utilisé le modèle de l'onde diffusante développé par Hayami, mais aucune référence n'ayant pu être trouvée sur l'utilisation de ce modèle dans le cas des réseaux d'assainissement, on a préféré ensuite adopter un modèle plus simple déjà largement employé dans ce cas, le modèle de Muskingum.

Ce modèle dont Cunge a montré qu'il constituait, dans certaines conditions, une bonne approximation de l'équation du second ordre d'Hayami, s'exprime par:

$Q_{s(t+\Delta t)}=C_{1} Q_{e(t)}+C_{2} Q_{e(t+\Delta t)}+C_{3} Q_{s(t)}$

$Q_{e}$ et $Q_{s}$ étant respectivement les débits d'entrée et de sortie et $C_{1}, C_{2}$, et $C_{3}$ des coefficients calculés de la manière suivante :

$$
\begin{aligned}
& C_{1}=\frac{K^{\prime} x+\Delta t / 2}{(1-x) K^{\prime}+\Delta t / 2} \\
& C_{2}=\frac{\Delta t / 2-K^{\prime} x}{(1-x) K^{\prime}+\Delta t / 2} \\
& C_{3}=\frac{K^{\prime}(1-x)-\Delta t / 2}{(1-x) K^{\prime}+\Delta t / 2}
\end{aligned}
$$

avec $K^{\prime}=L / c \quad L:$ longueur du tronçon $c$ : célérité de l'onde cinématique

et

$$
x=\frac{1}{2}\left(1-\frac{K^{\prime} Q_{0}}{L^{2} B I}\right)
$$

$B$ étant la largeur au miroir, $I$ la pente du tronçon et $Q_{0}$ le débit du régime permanent pour la profondeur d'écoulement.

\section{Propagation de la pollution}

Alors que le débit se propage à la vitesse de l'onde cinématique, les polluants dissous ou en suspension se déplacent plus lentement, sensiblement à la vitesse moyenne des molécules d'eau.

Pour calculer les flux polluants qui sortent du tronçon à partir des flux d'entrée dont l'évolution dans le temps est connue, il faut connaître, en sus des modifications de caratéristiques qui résultent d'éventuels dépôts ou remise en suspension de matières décantables, le temps moyen de transit ou ce qui est équivalent, la vitesse moyenne de transit de l'eau dans le tronçon.

Il s'agit là d'une vitesse moyenne sur la longueur du tronçon, dans le temps mis pour parcourir cette longueur, et ceci pour l'élément qui sort à un instant donné.

On la calcule dans FLUPOL de la manière suivante :

On détermine pour le temps de sortie choisi $N \cdot \Delta t$, le profil longitudinal du débit dans le tronçon. Il lui correspond un débit moyen $Q M(N)$ qui s'écrit :

$$
Q M(N)=\frac{1}{j \cdot \Delta \ell} \sum_{j=0}^{J} Q(j) \cdot \Delta \ell
$$

$\Delta \ell \quad$ la valeur du pas d'espace retenu pour le profil de débit;

$Q(J)$ la valeur du débit aux différents pas d'espace $J$; $J \quad$ le nombre total des pas d'espace.

A ce débit correspond, en régime permanent et uniforme, une vitesse moyenne $V M(N)$ (qui n'est pas, il faut le préciser, la grandeur que l'on veut déterminer).

On cherche alors le nombre de pas $N-N i$ pour lesquel la vitesse moyenne sur le temps de transit VMM1, correspondant au temps de sortie $N \cdot \Delta t$,

$$
V M M 1=\frac{1}{\left(N-N_{i}\right) \Delta t} \sum_{K=N}^{N-N_{i}} V M(K) \Delta t
$$

est égale à la vitesse $V M M 2$, rapport de la longueur du tronçon au temps mis par l'eau pour le parcourir:

$$
V M M 2=L /\left(N-N_{i}\right) \cdot \Delta t
$$

En d'autres termes et d'une manière plus analytique, tout cela revient à déterminer la borne supérieure $T i$ de l'intégrale double :

$\frac{1}{S_{m}\left(T_{s}-T_{i}\right) L} \int_{T_{s}}^{T_{i}} \int_{0}^{L} Q(\ell) \cdot d \ell \cdot d t$

de telle sorte que celle-ci égale $L /\left(T_{s}-T_{i}\right)$

avec

$L \quad$ Longueur du tronçon;

$S_{m}$ section transversale moyenne d'écoulement pour le débit longitudinal moyen au temps $T_{s}$;

$T_{s}$ temps de sortie pour lequel on cherche à connaitre la vitesse ou le temps de transit;

$T_{i}$ temps d'entrée de la pollution correspondant au temps de sortie $T_{s}$.

Connaissant le temps de transit $\left(N-N_{i}\right) \cdot \Delta t$, il est aisé de reconstituer les pollutogrammes de sortie à partir des pollutogrammes d'entrée dans le tronçon, grâce à un simple décalage dans le temps de ces derniers. Mais on ne tient pas compte ainsi des phénomènes de décantation ou de remise en suspension qui peuvent intervenir dans le tronçon considéré.

\section{Phénomènes de décantation ou de remise en suspension}

Selon les conditions de l'écoulement, les matières en suspension dont les effluents sont chargés risquent de sédimenter ou inversement des dépôts précédemment accumulés sur le fond des conduites peuvent être remis en suspension.

La prise en compte de ces phénomènes, absolument impérative dans certains cas et en particulier lorsque la pente des conduites est faible et qu'il existe des débits d'eaux usées de temps sec, pose un délicat problème de modélisation.

Cela ne résulte pourtant pas d'une absence de travaux sur la question, celle-ci se rencontrant également en hydraulique fluviale et ayant mobilisé au contraire l'intérêt de très nombreux chercheurs. 
Mais les spécialistes du transport solide considèrent généralement que ce dernier se décompose en deux phénomènes distincts et complémentaires le charriage ou saltation qui intervient à proximité du fond, et le transport en suspension qui se déroule au-dessus, la frontière entre les deux zones d'intervention n'étant d'ailleurs pas définie avec précision.

Si l'on considère toutes les implications de la prise en compte des phénomènes de sédimentation ou de remise en suspension, et en particulier la nécessité de traiter distinctement le cas des polluants décantables ou non, et celle de tenir un bilan, dans le temps, des dépôts présents sur le fond des conduites, on arrive vite à la conclusion qu'il serait très souhaitable pour ne pas ajouter à la complexité de l'ensemble d'utiliser un modèle aussi simple que possible et qui traduise globalement l'effet des deux phénomènes précités.

On peut pour cela faire appel à un modèle basé sur des considérations énergétiques qui a été attribué à Velikanov, mais sur lequel d'autres chercheurs ont également travaillé.

Une relation peut être établie entre les conditions hydrauliques d'un écoulement et la concentration de matières en suspension dans celui-ci.

Dans cette relation, la concentration de matières en suspension est fonction de la fraction d'énergie totale consommée pour maintenir ou pour répartir et maintenir les particules au sein du liquide. Selon Velikanov ces fractions d'énergie sont constantes.

Il faut donc distinguer deux niveaux d'énergie distincts et non un seul, conduisant chacun à une relation d'équilibre concentration-caractéristiques de l'écoulement.

On a ainsi

$$
\begin{aligned}
& C_{\text {MAX }}=\eta_{1} \frac{\rho_{s} \rho}{\rho_{s}-\rho_{e}} \cdot \frac{U}{W} \cdot \dot{J}_{p} \\
& C_{\text {MIN }}=\eta_{2} \frac{\rho_{s} \rho}{\rho_{s}-\rho_{e}} \cdot \frac{U}{W} \cdot \dot{J}_{p}
\end{aligned}
$$

$\rho_{s}$ et $\rho_{e}$ masses volumiques des particules et de l'eau;

$\rho \quad$ masse volumique de la suspension;

$U \quad$ vitesse moyenne d'écoulement;

W vitesse de chute des particules;

$J_{p} \quad$ perte de charge linéaire;

$\eta_{1}$ et $\eta_{2}$ sont les fractions d'énergie totale.

Dans FLUPOL on a retenu $\eta_{1}=0,022$ et $\eta_{2}=0,018$

Pour toute concentration comprise entre les deux valeurs d'équilibre la situation est stable et il n'y a ni décantation ni érosion. Si la concentration préexistante est supérieure à la concentration d'équilibre la plus élevée, il se produit une décantation jusqu'à ce que cette concentration d'équilibre soit atteinte; au contraire, si la concentration préexistante est inférieure à la concentration d'équilibre la plus faible, et s'il existe des dépôts, ceux-ci vont être érodés jusqu'à ce soit atteinte la concentration d'équilibre la plus basse.

Les particules en suspension entrainées dans les réseaux d'assainissement occupent une très large gamme granulométrique. Il serait tout à fait possible d'en tenir compte en répartissant les particules en un certain nombre de classes granulométrique et en procédant à des calculs séparés pour chacunes d'elles. Il n'a cependant pas paru très réaliste d'attendre d'une telle procédure un gain significatif de précision et on a supposé pour l'instant que les particules avaient toutes la même vitesse de chute, la valeur retenue relevant plus du calage que de considérations sur le diamètre (mal défini) des particules. Il a toutefois fallu faire une distinction entre les matières en suspension des eaux usées de temps sec et celles des eaux pluviales.

En outre les concentrations réellement atteintes après décantation ou érosion ont été obtenues en appliquant aux concentrations d'équilibre indiquées ci-avant un rendement, fonction dans le premier cas de la plus ou moins grande facilité de capture des particules en cours de décantation et, dans le second, de la plus ou moins grande facilité d'érosion des dépôts.

Il est ainsi possible de calculer pour chaque tronçon du réseau et pas de temps par pas de temps la concentration, donc le flux, des matières en suspension en sortie et la quantité de dépôts présente sur le fond des canalisations, ces dépôts fixant éventuellement de nouvelles conditions d'écoulement et conditionnant l'existence d'une sédimentation ou d'une érosion au pas de temps suivant.

Pour quantifier l'incidence des remises en suspension sur les flux polluants autres que celui des matières en suspension, il faut connaître la composition des solides déposés sur le fond des canalisations. celle-ci est naturellement différente de la composition des particules entraînées par le ruissellement ou des matières solides présentes dans les eaux usées urbaines.

Pour l'instant les travaux de calage déjà effectués conduisent à penser que les dépôts contiennent $10 \%$ de DBO5, $50 \%$ de DCO et $5 \%$ de NTK.

\section{Exemple d'utilisation}

Il est d'usage d'illustrer un article relatif à un modèle de simulation mathématique par un exemple d'utilisation permettant de juger de son domaine d'application et de comparer à des mesures effectuées préalablement, le résultat de simulations réalisées dans les mêmes conditions.

Pour être parfaitement convaincant, il faudrait même présenter plusieurs exemples comparatifs de ce genre, effectués sur des sites différents et dans des conditions de pluviométrie variables. Cela n'est pas possible dans le cadre d'un article ayant pour but la présentation générale du modèle et on se bornera ici à ne donner qu'un seul exemple; il est cependant envisagé de faire suivre ce premier article d'un second présentant plusieurs cas d'application et traitant de problèmes de calage.

L'exemple en question porte sur un collecteur pluvial de grande dimension de l'agglomération parisienne dénommé Fresnes-Choisy qui dessert dans la banlieue sud de Paris, une région importante des départements des Yvelynes, de l'Essonne, des Hauts de Seine et du Val de Marne. En fait, cet égout n'est pas strictement séparatif; il transite quelques eaux de temps sec consti- 
tuées d'eaux usées domestiques collectées dans certains tronçons amont du réseau ainsi que des eaux superficielles qui ont été dérivées dans l'égout. L'exutoire du réseau est la Seine, en rive gauche, à Choisy le Roi.

Seule la partie aval du réseau qui est située essentiellement dans le Val de Marne et qui dessert une zone de collecte de près de 2500 ha a fait l'objet d'une étude détaillée. Cette zone est représentée sur la figure 2.

Une campagne de mesures a été organisée en août, septembre et octobre 1986 afin de mesurer par temps de pluie les débits et les caractéristiques de pollution d'une part des effluents provenant des départements amont et entrant dans la zone d'étude, d'autre part des effluents sortant de cette zone et rejetés en Seine. Il est bon de signaler que des mesures ont simultanément été effectuées en Seine afin de pouvoir juger de l'impact du rejet du collecteur sur la qualité des eaux du fleuve.

L'organisation et l'exécution de ces deux campagnes ont été confiées au Laboratoire régional de l'ouest parisien.

Les caractéristiques pluviométriques ont pu être connues grâce aux enregistrements de quatre pluviographes que la direction départementale de l'Equipement du Val de Marne exploite dans le bassin de collecte à Rungis, Orly, Chevilly-la-Rue et Vitry-sur-Seine et qu'il faut remercier ici de sa très efficace coopération tant pour les données fournies que pour l'aide apportée à la mise en place du dispositif de mesures.

La période de mesures avait été choisie pour correspondre à des conditions météorologiques généralement orageuses et à un débit en Seine assez faible pour que des rejets d'eaux pluviales puissent nettement modifier les caractéristiques de l'eau du fleuve. Mais cette période n'a malheureusement pas été très pluvieuse : seuls trois événements pluvieux d'importance convenable ont pu être analysés.

C'est le premier d'entre eux, survenu le 22 août 1986 , après 37 jours de temps sec, qui a été retenu pour illustrer cet article. La totalité de la zone étudiée a été touchée par cet événement et les enregistrements des pluviographes ont montré que la pluie y a été homogène.

Avant d'être utilisé pour simuler les rejets de l'égout pluvial Fresnes-Choisy, le modèle FLUPOL avait fait l'objet d'un précalage à l'aide de résultats de mesures disponibles à l'Agence de bassin Seine-Normandie et notamment ceux acquis en 1981 lors de l'étude de deux bassins versants expérimentaux effectuée à Maurepas et aux Ulis sous l'égide du Service technique de l'urbanisme du Ministère de l'Equipement, du Logement, de l'Aménagement du territoire et des Transports.

Ce précalage ainsi que l'application du modèle au cas de l'égout pluvial Fresnes-Choisy ont été réalisés avec la très efficace collaboration de L. Herremans.

La zone d'étude a été découpée en 45 sous-zones de caractéristiques homogènes et le réseau d'assainissement a été limité, pour les simulations, aux canalisations d'un diamètre supérieur à $600 \mathrm{~mm}$ dont la longueur développée dépasse encore 23 kilomètres. A titre indicatif le diamètre de l'égout est de $4 \mathrm{~m}$ dans sa partie la plus aval.

Le degré de pollution du sol et la quantité de dépôts sur le fond des conduites à la fin de la pluie du 16 juillet qui a précédé celle du 22 août ont été supposés nuls, hypothèse plausible compte tenu de la forte intensité de

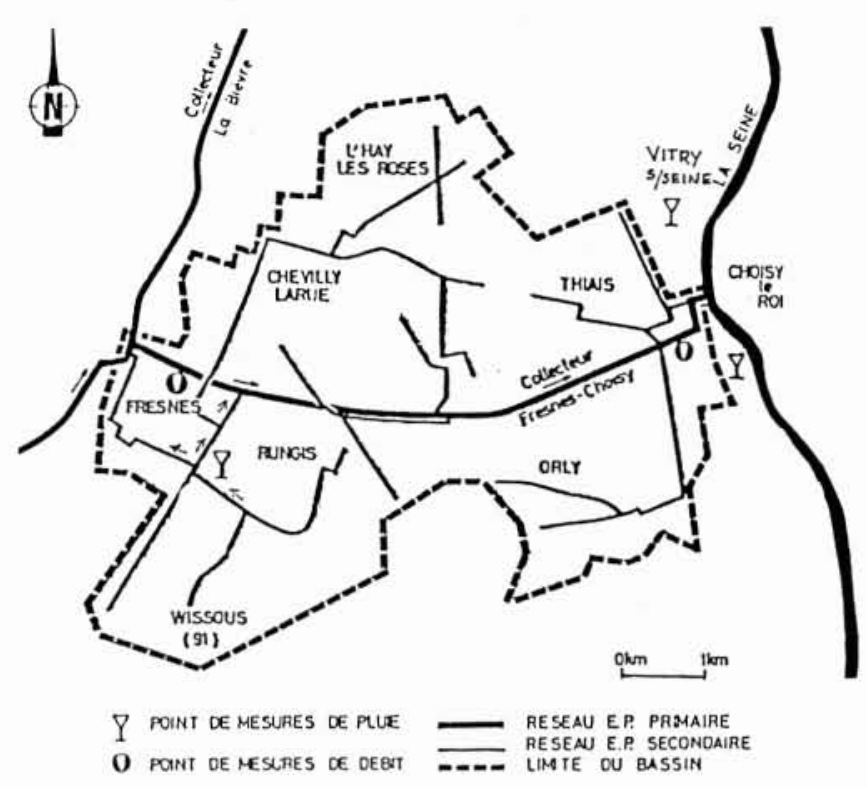

2. Zone de collecte modélisée.

cette pluie; de toute façon cette hypothèse ne peut avoir qu'une incidence limitée sur les résultats en raison de la très longue durée de temps sec entre les deux pluies (37 jours). S'il n'en avait pas été ainsi, il aurait fallu procéder à une simulation continue de toutes les pluies à partir d'un événement pluvieux antérieur suffisamment intense. C'est par exemple ce qui a été fait pour simuler les deux autres pluies importantes survenues au cours de la campagne de mesures.

La figure 3 (page suiv.) présente graphiquement l'évolution dans le temps des caractéristiques de la pluie et de l'effluent à l'exutoire du réseau. La représentation sur les mêmes graphiques, tant pour le débit que pour les divers paramètres de pollution des courbes des valeurs mesurées et calculées permet de juger de l'aptitude du modèle à simuler correctement la réalité. Ce résultat n'est pas fortuit; les deux autres pluies importantes de la campagne ont pu être simulées avec une précision équivalente, sans qu'il soit nécessaire de modifier le programme de calcul ou la valeur des coefficients numériques.

La part des eaux de temps sec à la sortie de l'égout est très faible, $75 \mathrm{l} / \mathrm{s}$ pour le débit, environ $10 \mathrm{~g} / \mathrm{s}$ pour les MES et la DCO et $5 \mathrm{~g} / \mathrm{s}$ pour la DBO5.

Les charges globales évacuées par le réseau à l'occasion de cet événement pluvieux sont données dans le tableau de la page suivante, qui montre que l'écart par rapport aux mesures n'excède pas $15 \%$, celui-ci étant constaté pour la DBO5 dont on sait que le dosage est particulièrement délicat. 

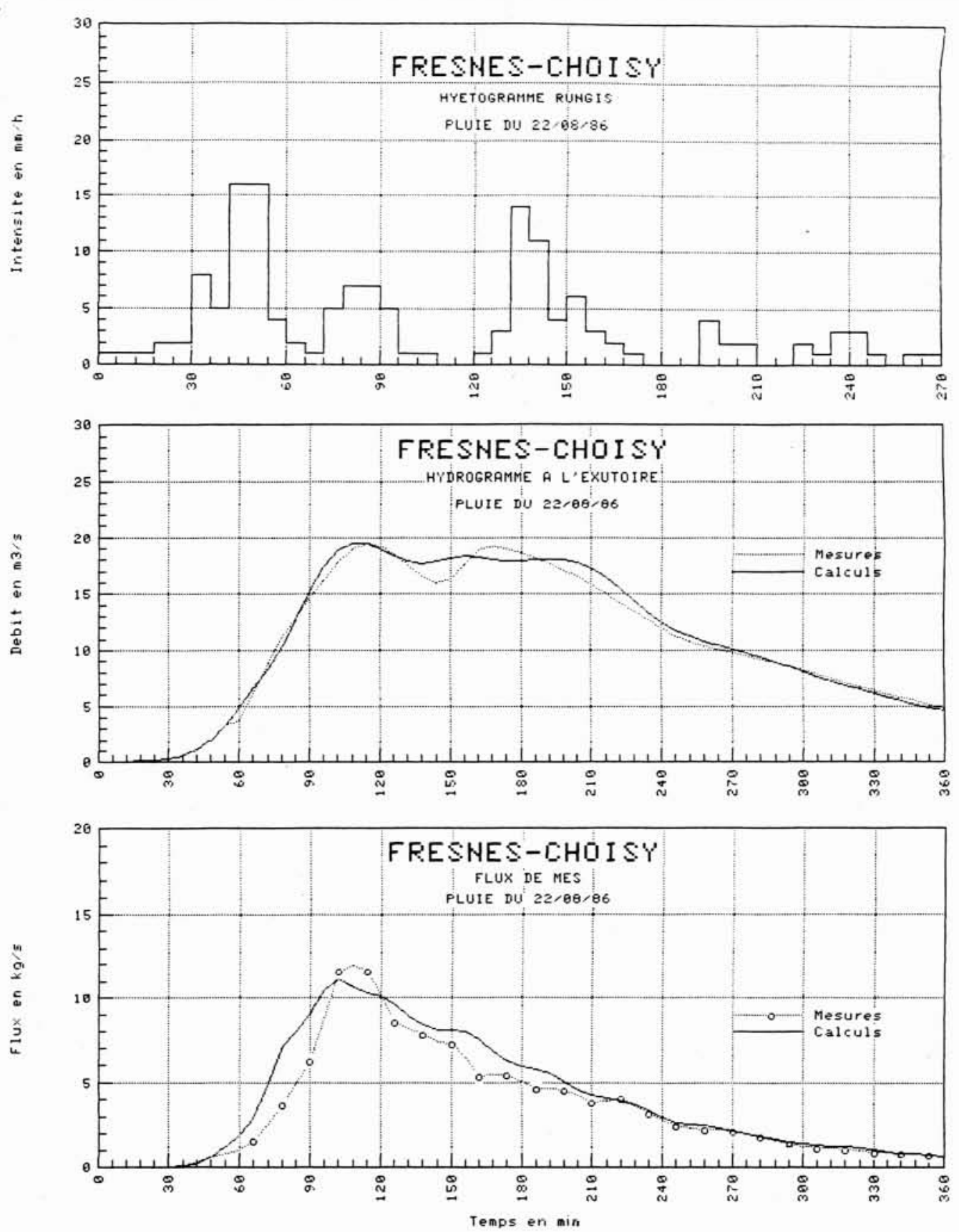

3.

Pluie du 22-08-1986. Charges totales mesurées et simulées à l'exutoire du collecteur Fresnes-Choisy.

\begin{tabular}{|l|c|c|c|}
\hline & Mesures 1 & Simulation 2 & Rapport 2/1 \\
\hline $\begin{array}{l}\text { Volume } \\
\left(\mathrm{m}^{3}\right)\end{array}$ & 239138 & 237797 & 1 \\
\hline $\begin{array}{l}\text { Masse MES } \\
(\mathrm{kg})\end{array}$ & 77720 & 86942 & 1,12 \\
\hline $\begin{array}{l}\text { Masse DBO5 } \\
(\mathrm{kg})\end{array}$ & 7417 & 8427 & 1,14 \\
\hline $\begin{array}{l}\text { Masse DCO } \\
(\mathrm{kg})\end{array}$ & 39643 & 41565 & 1,05 \\
\hline $\begin{array}{l}\text { Masse NTK } \\
(\mathrm{kg})\end{array}$ & 1363 & 1535 & 1,13 \\
\hline
\end{tabular}

\section{Conclusion}

Au terme de cette présentation plutôt favorable le lecteur pourrait penser que le modèle FLUPOL a maintenant atteint une forme définitive. Ce serait méconnaître la démarche longue et laborieuse par laquelle s'affine un modèle mathématique ainsi que la mise au point d'un programme de calcul informatisé. Les 289 précédentes versions de FLUPOL sont là pour illustrer ces propos.

Il n'y a pas en effet de progrès en matière de simulation sans une confrontation continuelle du modèle et de la réalité. Cette confrontation peut conduire à la remise en cause de certains algorithmes ou à la prise en 

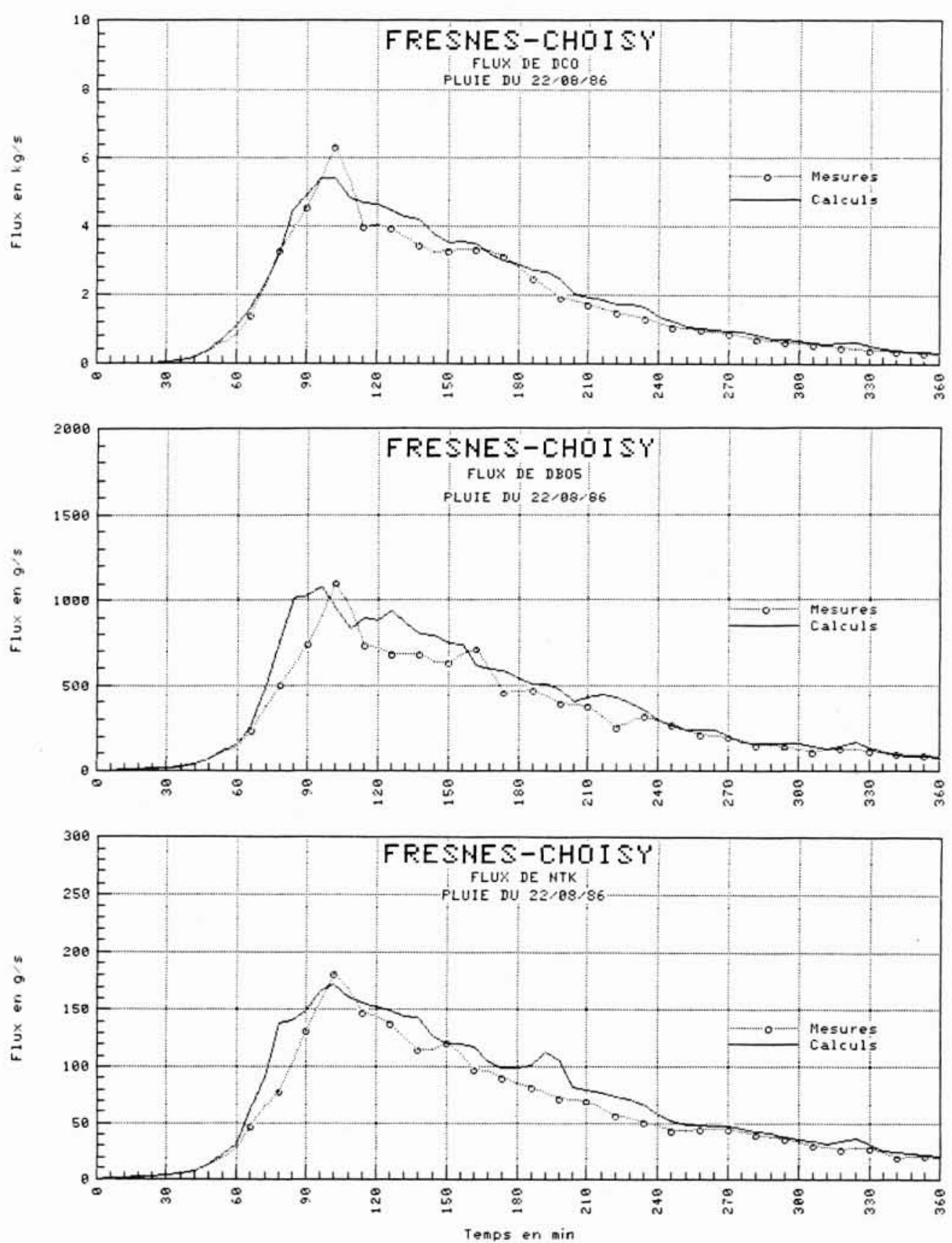

compte de phénomènes jusqu'alors négligés; elle peut aussi susciter des élargissements du domaine d'application de l'ensemble.

Ces corrections, transformations ou améliorations peuvent avoir des conséquences indésirables, difficilement prévisibles et il faut vérifier que leur mise en œuvre n'infirme pas les résultats satisfaisants obtenus antérieurement. C'est là une des raisons pour lesquelles la difficulté de mise au point croît avec son degré d'avancement.

Mais il ne faut pas verser dans le perfectionnisme et oublier, le mieux étant l'ennemi du bien, qu'il existe un réel besoin de modèles du type FLUPOL. Il paraît donc judicieux de procéder par vérités provisoires. S'il est certainement possible de mieux faire, le modèle actuel donne, en tout état de cause, dès à présent des résultats convenables eu égard à l'objectif visé, c'est-à-dire la gestion de l'environnement.

D'ailleurs une utilisation pertinente d'un modèle de simulation est souvent bien plus importante que son niveau de sophistication qui, au-delà de certaines limites, peut paraître un peu illusoire. Cette réflexion conduit à souligner le rôle déterminant du personnel chargé de l'exécution des simulations qui devrait toujours être choisi parmi des techniciens expérimentés et avertis de toutes les particularités des modèles qu'ils exploitent. 


\section{Liste des symboles}

$a, b . d$
$A$
$A R$
$B$
$c$
$C_{1}, C_{2}, C_{3}$
$C_{\mathrm{MAX}}$
$C_{\mathrm{MIN}}$
$D E N S$
$D I$
$F R$
$F_{i}$
$F(t)$

$G P$
$H$
$H P E$
$I$
$i$
$I M P R$
$I M A X$
$J$
$J p$
$K$
$K^{\prime}$
$K A, K a^{\prime}$
$K 1$
$K P$
$L$
$L D E P$
$L N G$
$M$
$M e(t)$

MO

$M R$

$M R O$

$P N T$

PS

$P T E$

$Q$

$Q_{e}$

$Q_{i}$

$Q(j), Q(\ell)$

$Q M(N)$

Q。

$Q_{s}$

QSEC

$Q U$

$S A F$

$S E$

$\mathrm{Sm}$

$t$

T

T3

$T_{i}, T_{s}$

$u$ VIT, $U$ $V M(N)$
Coefficients numériques intervenant dans le calcul de $K P$

Coefficient de correction des masses de polluants solides entrainées par la pluie

Surface du bassin versant homogène

Largeur de l'écoulement au miroir

Célérité de l'onde cinématique

coefficients du modèle de Muskingum

Concentration d'équilibre en cas de sédimentation

Concentration d'équilibre en cas d'érosion

Densité de population d'un sous bassin

Diamètre d'une conduite circulaire

Fraction de population raccordée au réseau

Flux résiduel de $M E S$ d'une pluie précédente au temps $O$, rapporté à l'unité de surface $\mathrm{kg} / \mathrm{ha} . \mathrm{s}$ Flux de matières en suspension à la sortie d'un $\mathrm{S} /$ bassin homogène rapporté à l'unité de surface

Charge polluante spécifique des eaux usées

hauteur d'eau dans une canalisation rectangulaire

$\mathrm{kg} / \mathrm{ha} . \mathrm{s}$

Hauteur d'eau précipitée

Pente d'un tronçon homogène

Intensité de la pluie

Coefficient d'imperméabilisation

Intensité max de la pluie sur $5 \mathrm{mn}$

$\mathrm{kg} / \mathrm{hab} . \mathrm{j}$

$\mathrm{m}$

m

$\%$

$\mathrm{m} / \mathrm{h}$

Nombre total de pas d'espace

Perte de charge linéaire

Coefficient de proportionnalité entre volume stocké et débit sortant ou entre masse

stockée en suspension et flux sortant

Coefficient intervenant dans le modèle de Muskingum

Coefficients d'élimination des polluants solides du sol par la pluie

Fraction en poids d'un polluant donné dans le

Longueur d'une canalisation

Largeur des dépôts en surface

Long max. d'un sous bassin homogène

Masse de polluants solides déposée sur le sol par temps sec

$\mathrm{mm} / \mathrm{h}$

$-$

Masse de solides mis en suspension dans l'eau de ruissellement au temps $t$ et rapportée à l'unité de surface

Masse de polluants solides au sol au début de la pluie

Masse de polluants solides au sol au temps $t$ ou en fin d'un pas de temps $\Delta t \quad \mathrm{~kg}$

Masse de polluants solides au sol au début d'un pas de temps $\quad \mathrm{kg}$

Pente moyenne du terrain

Masse de poussières et salissures se déposant au sol dans l'unité de temps et d'espace $\mathrm{kg} / \mathrm{ha}$.j

Coefficient de pointe horaire des eaux usées de temps sec

Débit du réseau d'assainissement rapporté à l'unité de surface

Débit d'entrée d'un tronçon homogène

Débit résiduel d'une pluie précédente au temps zéro rapporté à l'unité de surface

Débits au pas d'espace $j$ ou $l$

Débit moyen de l'hydrogramme au pas $N$

Débit du régime permanent correspondant à la profondeur de l'écoulement

Débit de sortie d'un tronçon homogène

Débit de temps sec

Rejet spécifique d'eaux usées de temps sec

Surface transversale à l'écoulement occupée par des dépôts

Surface transversale à l'écoulement occupée par de l'eau

$\mathrm{m} / \mathrm{s}$

$\mathrm{m}^{3} / \mathrm{s}$

$\mathrm{m} / \mathrm{s}$

$\mathrm{m}^{3} / \mathrm{s}$

$\mathrm{m}^{3} / \mathrm{s}$

$\mathrm{m}^{3} / \mathrm{s}$

$\mathrm{m}^{3} / \mathrm{s}$

$\mathrm{m}^{3} / \mathrm{s}$

$\mathrm{m}^{3} / \mathrm{jxhab}$

$\mathrm{m}^{2}$

$\mathrm{m}^{2}$

Section transversale moyenne d'écoulement pour le débit longitudinal moyen au temps $T S$

Temps

Temps d'intégration

Durée de la pluie

Temps d'entrée et de sortie de l'eau d'un tronçon

Temps courant depuis le début de la pluie

Vitesse moyenne d'écoulement de l'eau

Vitesse moyenne de l'eau au pas $N$ 
VMM1, VMM2

$W$

$W A, W B$

$\Delta t$

$\Delta \ell$

$\phi e(t)$

$\eta 1, \eta 2$

$\rho_{s}, \rho_{e}, \rho$
Vitesses moyennes pour l'eau sortant du tronçon au temps $N . \Delta t$

$\mathrm{m} / \mathrm{s}$

Vitesse de chute des particules solides

$\mathrm{m} / \mathrm{s}$

Variables intermédiaires dans le calcul de $c$

$-$

$\mathrm{s}$

$\mathrm{m}$

pas d'espace

Flux de particules solides évacué d'un sous bassin homogène rapporté à l'unité de surface

Fractions d'énergie totale consommée par l'écoulement

Masses volumiques des particules solides, de l'eau et de la suspension $\mathrm{kg} / \mathrm{ha} . \mathrm{s}$

$\mathrm{kg} / \mathrm{m}^{3}$

\section{Bibliographie}

[1] Desbordes M. - Réflexion sur les méthodes de calcul des réseaux urbains d'assainissement pluvial.

Université des sciences et techniques du Languedoc-Montpellier.

Thèse de Docteur-Ingénieur - 224 pages - février 1974.

[2] Desbordes M. - Un essai de modélisation des phénomènes de ruissellement pluvial urbain.

Techniques et Sciences Municipales - pages 121-126 mars 1975.

[3] Hemain J.C. (L.H.M.). - Etude bibliographique des modèles de qualité des eaux drainées par les réseaux d'assainissement urbains.

Ministère de l'Environnement et du Cadre de Vie Service technique de l'urbanisme, Paris (15) - 246 pages - septembre 1979.

[4] Hemain J.C. (L.H.M.). - Mesure de la pollution du ruissellement pluvial urbain - Suivi et bilan de la cam. pagne expérimentale,

Ministère de l'Urbanisme et du Logement - Service technique de l'urbanisme Paris (15) - 74 pages - juillet 1983.
[5] Sutherland R.C., Mac Cuen R.M. - Simulation of urban no-point source pollution.

AWRA Water Resources Bulletin - Vol 14, $\mathrm{n}^{\circ} 2-1978-$ pages 409-428.

[6] Combes V. - Etude de modèles mathématiques de transport de matériaux solides en réseaux d'assainissement. Institut national polytechnique de Toulouse - D.E.A. de mécanique - 153 pages - juillet 1982.

[7] WIUfF RASMUS. - Transport of suspended material in open and submerged streams.

Journal of Hydraulic Engineering, Vol 111, No 5 - ma 1985 - 18 pages.

[8] LARRAS J. - Hydraulique et granulats. Eyrolles - Paris - 254 pages - 1972.

[9] Ranchet J. et Deutsch J.C. - Caractérisation de la pollution des eaux de ruissellement en milieu urbain. Techniques et Sciences Municipales - pages 385-394 août-septembre 1982.

[10] Grange D. et al. - Mesure de l'impact des eaux pluviales d'un grand bassin versant urbain sur la Seine: cas du bassin du collecteur Fresnes-Choisy.

A.F.B.S.N. - L.R.O.P. 45 p. + annexes. Juin 1987. 